\title{
Variabilité de la reproduction examinée au laboratoire entre populations naturelles d'Helix aspersa Müller de la région Bretagne
}

\author{
L Madec, J Daguzan \\ LA INRA, Laboratoire de zoologie générale d'écophysiologie, \\ Av du Général Leclerc, 35042 Rennes Cedex, France
}

(Reçu le 18 juin 1990; accepté le 24 juin 1991)

\begin{abstract}
Résumé - Les effets de la localisation géographique des populations naturelles et de la date de la collecte sur la reproduction de l'escargot "petit-gris" Helix aspersa Müller ont été recherchées en conditions artificielles normalisées. En Bretagne, les variations observées entre populations traduisent la flexibilité des caractères liés à l'activité reproductrice et la relative stabilité des capacités reproductrices.
\end{abstract}

gastéropode terrestre / reproduction / variabilité géographique

Summary - Reproductive variability in laboratory conditions between natural populations of Helix aspersa Müller of the region Brittany. Laboratory experiments were conducted to investigate the effects of geographical localization of natural populations and sampling dates on the reproduction of the garden snail Helix aspersa Müller. In Brittany, reproductive variability between populations shows the adaptive flexibility of characters describing reproductive activity and the relative stability of reproductive ability.

terrestrial gastropod / reproduction / geographic variability

\section{INTRODUCTION}

Helix aspersa Müller est un Gastéropode pulmoné dont l'origine semble être le bassin méditerranéen occidental (Sacchi, 1958; Peake, 1978). Les étapes ultérieures de sa diffusion, étroitement tributaires des déplacements et des activités de l'homme, ont élargi cette aire de répartition initiale puisque l'espèce est au- jourd'hui implantée sur 4 continents. La diversité des milieux colonisés a comme conséquence l'expression de phénotypes particuliers illustrant l'adaptabilité de la morphologie, de la physiologie et de l'éthologie de l'espèce; ainsi, la variabilité de son cycle biologique se traduit essentiellement par des ajustements saisonniers liés à la latitude (Ingram, 1946, Potts, 1975; Moulin, 1980; Chevallier, 
1980; Crook, 1980). Des recherches ont permis de connaître l'origine et d'apprécier l'amplitude de la variabilité des principales caractéristiques de la reproduction au sein d'une même population d'Helix aspersa (Madec et Daguzan, 1987; Madec, 1989); elles montrent que l'activité reproductrice (accouplement, ponte) est influencée par certains facteurs de l'environnement tandis que la taille de la ponte, corrélée à la taille des individus, paraît moins dépendante des conditions extérieures.

Ces observations démontrent l'intérêt d'une étude comparative précise de la reproduction d'escargots issus de populations soumises, en milieu naturel, à des contraintes écologiques très variées. La comparaison rapportée dans la présente étude a été réalisée sur 5 échantillons provenant d'une seule région, mais prélevés dans des milieux différents au printemps des années 1983 et 1986.

\section{MATÉRIEL ET MÉTHODES}

\section{Échantillonnage et conditionnement des escargots}

Les sites de prélèvement sont situés à Lorient et Larmor (Morbihan), Rennes et Paimpont (Illeet-Vilaine), Scaër (Finistère). Seule la station de Larmor, située au niveau de la lisière sablonneuse littorale, présente un sol relativement riche en calcaire (maërl, débris coquilliers). Dans tous les milieux, on trouve en abondance du lierre (Hedera helix Linné), des ronces (Rubus sp), de l'ortie (Urtica sp) et de nombreuses espèces de Graminées.

Les données mésoclimatiques relevées dans les stations météorologiques les plus proches sont présentées dans le tableau 1 (indice de Martonne) : elles indiquent une pluviométrie croissante de l'Est vers l'Ouest de la Bretagne.

Les escargots sont récoltés en fin d'hibernation, sur une surface ou une longueur limitée (tableau I). Ils sont ensuite conservés dans cet état physiologique à l'obscurité complète dans une chambre froide $\left(\mathrm{T}^{\circ}=5 \pm 1{ }^{\circ} \mathrm{C}\right.$; Humidité relative : $\mathrm{Hr}=80 \pm 5 \%$; Jour/nuit : $L / D: 0 / 24 \mathrm{~h}$ ) avant d'être mesurés (grand diamètre de la coquille), numérotés puis placés pendant une semaine dans une pièce climatisée $\left(T^{\circ}=15\right.$ $\pm 1{ }^{\circ} \mathrm{C} ; \mathrm{Hr}=80 \pm 5 \%$; LD : 12/12 h) où ils reprennent progressivement leur activité (rupture de l'épiphragme, locomotion, alimentation).

Le protocole d'étude de la reproduction fait appel aux travaux de Daguzan (1981) sur des escargots soumis, en milieu naturel, à un climat de type océanique. Après la phase de conditionnement, les animaux sont introduits dans la salle destinée à la reproduction $\left(\mathrm{T}^{\circ}=20 \pm 1^{\circ} \mathrm{C}\right.$; $\mathrm{Hr}=80 \pm 5 \% ; \quad L \mathrm{D}: 16 / 8 \mathrm{~h}$ ). Les échantillons sont divisés en lots de 35 individus dans des enceintes de 0,5 $\times 0,3 \mathrm{~m}$, soit 234 individus par $\mathrm{m}^{2}$ au sol, représentant une charge biotique proche de la valeur optimale de $20 \mathrm{~kg} / \mathrm{m}^{3}$ préconisée pour la reproduction en conditions artificielles (Daguzan; 1981). Outre une mangeoire et une plaque de mousse synthétique saturée d'eau, chaque enceinte renferme 4 pots d'argile type "pépiniériste" contenant du terreau horticole stérilisé utilisé comme substrat de ponte.

\section{Observations et mesures}

Du fait de la durée de l'accouplement (environ $7 \mathrm{~h}$ ) et de celle de la ponte (environ $18 \mathrm{~h}$ ), on effectue 2 observations par jour ( $8-9 h ; 17-18 h$ ) sur une période qui s'étend du réveil des escargots jusqu'à l'arrêt de toute activité reproductrice. On note la date de reprise de l'activité de chaque individu, son comportement de reproduction ainsi que la mortalité. Dès qu'un escargot est observé en position de ponte, le pot le contenant est retiré, tout en laissant l'escargot terminer sa ponte, et immédiatement remplacé.

Les résultats sont exprimés en taux d'accouplement et de ponte (définis comme le pourcentage des individus s'accouplant et celui des individus pondant par rapport au nombre total d'escargots placés en reproduction), en nombres moyens $(m \pm$ sem) d'accouplements et de pontes par individu et en nombre d'œufs par ponte et par individu.

Avant toute comparaison, il faut s'assurer qu'il n'existe pas d'hétérogénéité entre les 
Tableau I. Caractéristiques écologiques des localités échantillonnées en Bretagne.

\begin{tabular}{|c|c|c|c|c|c|}
\hline \multirow{2}{*}{$\begin{array}{l}\text { Département } \\
\text { Commune }\end{array}$} & \multicolumn{2}{|c|}{ Ille-et-Vilaine } & \multicolumn{2}{|c|}{ Morbihan } & \multirow{2}{*}{$\begin{array}{c}\text { Finistère } \\
\text { Scaër }\end{array}$} \\
\hline & Rennes & Paimpont & Lorient & Larmor & \\
\hline Date de la récolte & 29 mars 83 & 16 avril 86 & 28 mars 83 & 15 avril 86 & 28 mars 83 \\
\hline Nature de l'habitat & $\begin{array}{c}\text { Jardin } \\
\left(200 \mathrm{~m}^{2}\right) \\
\text { Milieu fermé }\end{array}$ & $\begin{array}{c}\text { Jardin } \\
\left(5000 \mathrm{~m}^{2}\right) \\
\text { Migrations } \\
\text { possibles }\end{array}$ & $\begin{array}{l}\text { Talus en bordure } \\
\text { de champs } \\
\text { cultivés }\end{array}$ & $\begin{array}{l}\text { Lisière littorale } \\
\text { Biotope riche } \\
\text { en calcaire }\end{array}$ & $\begin{array}{l}\text { Friche à proximité } \\
\text { d'une ferme }\end{array}$ \\
\hline Type de colonie & Étalé & Étalé & Linéaire & Linéaire & Linéaire \\
\hline $\begin{array}{l}\text { Caractéristiques } \\
\text { géologiques }\end{array}$ & $\begin{array}{l}\text { Schistes } \\
\text { briovériens }\end{array}$ & $\begin{array}{c}\text { Grès } \\
\text { armoricain }\end{array}$ & Gneiss & Granulite & Micaschiste \\
\hline \multicolumn{6}{|l|}{$\begin{array}{l}\text { Indice d'aridité } \\
\text { de Martonne }\end{array}$} \\
\hline $\begin{array}{l}\text { - de l'année } \\
\text { - de juillet }\end{array}$ & \multicolumn{2}{|c|}{$\begin{array}{l}30 \\
17,5\end{array}$} & \multicolumn{2}{|c|}{$\begin{array}{l}43 \\
19,4\end{array}$} & $\begin{array}{l}64 \\
27,5\end{array}$ \\
\hline
\end{tabular}

échantillons pour les variables ayant une influence significative sur le nombre d'œufs par ponte, c'est-à-dire, en conditions artificielles, la taille des individus et le nombre de pontes par individu. On sait en effet que la taille de la ponte augmente avec celle du pondeur et que le nombre d'œufs déposés par un même individu lors de la seconde et la troisième ponte est inférieur à celui de sa première ponte (Madec et $\mathrm{Da}$ guzan, 1987).

Le traitement statistique des résultats repose sur les tests classiques de comparaison de 2 ou plusieurs échantillons. Pour les données quantitatives, on effectue des comparaisons de moyennes (test $t$, modifié ou non; analyse de variance) quand les paramètres des distributions le permettent ou, le cas échéant, des tests de comparaison non paramétriques (tests de Wilcoxon-Mann-Withney; test de Kruskal-Wallis). Pour les données qualitatives, les tableaux de contingence sont traités grâce au test $\chi^{2}$. Lorsqu'une hétérogénéité apparaît au sein d'une série d'échantillons, l'emploi d'un test de comparaisons multiples permet de préciser la signification des différences observées puis de classer les échantillons considérés.

\section{RÉSULTATS}

\section{L'activité reproductrice}

\section{Comparaison des fluctuations observées au laboratoire}

En 1983, la reprise de l'activité d'accouplement est immédiate dans les 3 populations d'escargots (fig 1) (différences entre échantillons non significatives). La reprise des pontes est observée à partir de 4-6 semaines; elle est plus précoce dans les lots de Lorient $(P=0,03)$. Cependant, si la différence entre échantillons les plus précoces (Lorient; Rennes) est significative pour les 5 premières pontes $(P=0,02)$, elle ne l'est plus pour les 10 premières.

En 1986, la reprise d'activité est beaucoup plus tardive; les premiers accouplements ne sont observés qu'à 6 semaines 

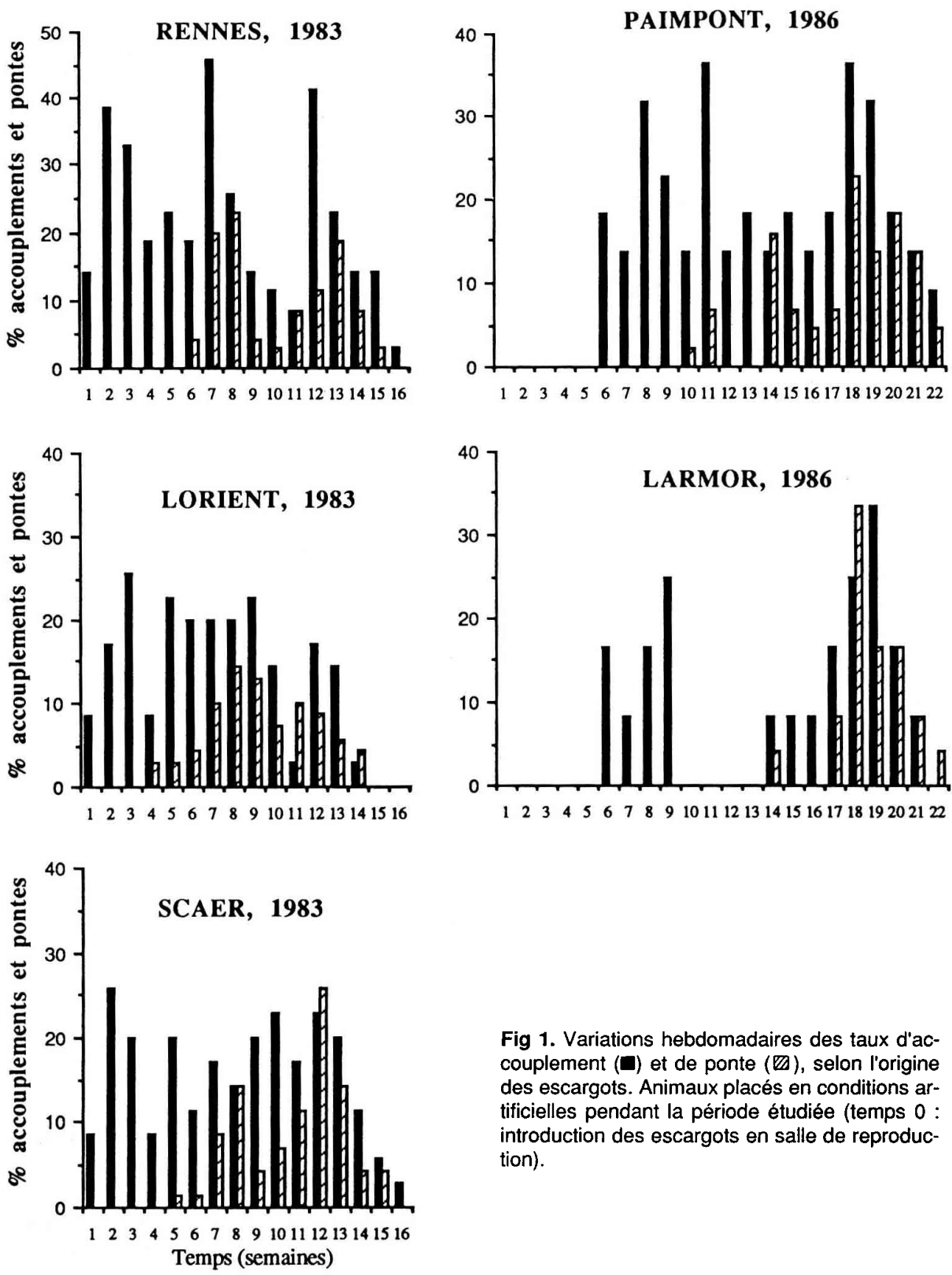

Fig 1. Variations hebdomadaires des taux d'accouplement $(\square)$ et de ponte ( $\square$ ), selon l'origine des escargots. Animaux placés en conditions artificielles pendant la période étudiée (temps 0 : introduction des escargots en salle de reproduction). 
et la reprise des pontes n'intervient qu'à 10 et 14 semaines selon les populations (fig 1).

L'évolution des nombres d'accouplements au cours de la période de reproduction étudiée en 1983 montre un rythme d'activité voisin pour tous les échantillons. La distribution des accouplements n'est pas statistiquement différente entre les lots. II en va différemment pour le nombre de pontes où l'échantillon du Morbihan présente un profil unimodal alors qu'il est bimodal pour les 2 autres groupes (différence très hautement significative entre les 3 distributions : $P=0,001$ ).

En 1986, l'activité d'accouplement des 2 échantillons suit un rythme bimodal avec 2 maximums, l'un vers 8-11 semaines et l'autre vers 18-19 semaines. L'activité de ponte ne présente quant à elle qu'un seul maximum lors de la $18^{\mathrm{e}}$ semaine (fig 1 ).

\section{Intensité de l'activité reproductrice}

Les taux moyens d'accouplement sont comparables, exception faite pour l'échantillon de Rennes 1983 chez lequel ce taux est très élevé (tableau II). L'activité d'accouplement est plus intense dans les échantillons de Rennes et de Paimpont où les individus s'accouplent en moyenne 3,4 fois contre seulement 1,9 à 2,5 fois dans les autres échantillons. La comparaison globale des nombres d'accouplements par individu (fig 2) se traduit par une différence très hautement significative $(P<0,001)$, alors que les variations observées entre échantillons de même origine mais constitués à des dates différentes semblent plutôt imputables aux fluctuations d'échantillonnage $(P>0,05)$. Sachant qu'un seul accouplement est souvent insuffisant pour assurer à chaque partenaire l'obtention d'un stock de spermatozoïdes, on remarque que les escargots ne s'étant accou- plés au plus qu'une seule fois sont environ 2 fois moins nombreux dans les lots de Rennes et Paimpont que dans les autres. Malgré ces variations du taux d'accouplement, il n'y a pas de différence significative entre échantillons du nombre moyen de pontes par escargot (tableau II).

\section{Les capacités reproductrices}

La taille des individus présente des différences très hautement significatives entre échantillons $(P<0,001)$ mais ne semble pas plus dépendre de l'origine géographique que de l'année de la collecte des escargots (tableau II). En revanche, le nombre de pontes par individu ne varie pas significativement d'un échantillon à l'autre $(P=0,43)$. Les comparaisons ultérieures s'appuient donc sur un nombre d'œufs par ponte calculé à partir de l'ensemble des pontes de chaque échantillon.

Les comparaisons des nombres moyens d'œufs par ponte et par individu permettent de conclure à l'homogénéité des moyennes considérées $(P>0,05)$. Pour expliquer la valeur modeste du résultat relatif à l'échantillon Paimpont 1986 compte tenu de la taille des individus qui le constituent, on peut rappeler que le nombre d'œufs par ponte est calculé à partir de toutes les pontes déposées or, $20,5 \%$ des animaux de cet échantillon ont pondu 2 fois, $11,4 \% 3$ fois avec une deuxième et une troisième ponte dont le nombre d'œufs est très inférieur à celui de la première. Ce phénomène contribue donc à une baisse plus sensible du nombre moyen d'œuts par ponte sur cet échantillon. De plus, la régression du nombre d'œufs par ponte $(N)$ en fonction du grand diamètre de la coquille $(D)$ calculée d'après les résultats de l'année 1983 $\left(N=0,73 D-122,6 ; R^{2}=0,26 ; P<0,001\right)$, laisse apparaître un déficit en œufs lors- 
Tableau II. Activité et capacités reproductrices obtenues en conditions artificielles, selon l'origine et l'année de récolte des escargots reproducteurs.

\begin{tabular}{|c|c|c|c|c|c|}
\hline \multirow[t]{2}{*}{ Origine } & \multicolumn{2}{|c|}{ Ille-et-Vilaine } & \multicolumn{2}{|c|}{ Morbihan } & \multirow{2}{*}{$\begin{array}{l}\text { Finistère } \\
\text { Scaër } \\
\text { (1983) }\end{array}$} \\
\hline & $\begin{array}{l}\text { Rennes } \\
\text { (1983) }\end{array}$ & $\begin{array}{l}\text { Paimpont } \\
\text { (1986) }\end{array}$ & $\begin{array}{c}\text { Lorient } \\
\text { (1983) }\end{array}$ & $\begin{array}{c}\text { Larmor } \\
(1986)\end{array}$ & \\
\hline Effectif initial & 70 & 70 & 70 & 35 & 70 \\
\hline Effectif de reproducteurs & 70 & 44 & 70 & 24 & 70 \\
\hline $\begin{array}{l}\text { Grand diamètre } \\
\text { de la coquille }(\mathrm{mm}) \text { * }\end{array}$ & $29,0 \pm 0,2$ & $31,2 \pm 0,2$ & $30,5 \pm 0,2$ & $32,1 \pm 0,3$ & $30,4 \pm 0,2$ \\
\hline $\begin{array}{l}\text { Taux moyen } \\
\text { d'accouplements (\%) }\end{array}$ & 92,9 & 79,5 & 80,0 & 79,2 & 84,3 \\
\hline $\begin{array}{l}\text { Nombre moyen d'accouplements * } \\
\text { - par individu } \\
\text { - par individu s'étant accouplé }\end{array}$ & $\begin{array}{l}3,4 \pm 0,2 \\
3,7 \pm 0,2\end{array}$ & $\begin{array}{l}3,4 \pm 0,3 \\
4,3 \pm 0,3\end{array}$ & $\begin{array}{l}2,1 \pm 0,2 \\
2,7 \pm 0,2\end{array}$ & $\begin{array}{l}1,9 \pm 0,3 \\
2,3 \pm 0,2\end{array}$ & $\begin{array}{l}2,5 \pm 0,5 \\
3,0 \pm 0,2\end{array}$ \\
\hline Taux moyen de pontes (\%) & 71,4 & 72,7 & 60,0 & 58,3 & 71,4 \\
\hline $\begin{array}{l}\text { Nombre moyen de pontes * } \\
\text { - par individu } \\
\text { - par individu ayant pondu }\end{array}$ & $\begin{array}{l}1,0 \pm 0,1 \\
1,5 \pm 0,1\end{array}$ & $\begin{array}{l}1,2 \pm 0,1 \\
1,6 \pm 0,1\end{array}$ & $\begin{array}{l}0,9 \pm 0,1 \\
1,5 \pm 0,1\end{array}$ & $\begin{array}{l}0,9 \pm 0,1 \\
1,5 \pm 0,1\end{array}$ & $\begin{array}{l}1,0 \pm 0,1 \\
1,4 \pm 0,1\end{array}$ \\
\hline Nombre moyen d'œufs par ponte * & $90,1 \pm 6,2$ & $93,4 \pm 5,8$ & $99,3 \pm 4,3$ & $108,7 \pm 5,7$ & $102,9 \pm 4,9$ \\
\hline $\begin{array}{l}\text { Nombre moyen d'œufs * } \\
\text { - par individu } \\
\text { - par individu ayant pondu }\end{array}$ & $\begin{array}{r}94 \pm 10 \\
132 \pm 10\end{array}$ & $\begin{array}{l}108 \pm 12 \\
149 \pm 11\end{array}$ & $\begin{array}{r}82 \pm 10 \\
141 \pm 10\end{array}$ & $\begin{array}{r}95 \pm 9 \\
163 \pm 9\end{array}$ & $\begin{array}{l}100 \pm 11 \\
140 \pm 12\end{array}$ \\
\hline $\begin{array}{l}\text { Mortalité (\%) } \\
\text { - avant reproduction } \\
\text { - en cours de reproduction }\end{array}$ & $\begin{array}{r}0 \\
24\end{array}$ & $\begin{array}{l}37 \\
54\end{array}$ & $\begin{array}{r}0 \\
27\end{array}$ & $\begin{array}{l}31 \\
43\end{array}$ & $\begin{array}{r}0 \\
24\end{array}$ \\
\hline
\end{tabular}

- $m \pm$ écart type à la moyenne.

qu'on y introduit les diamètres moyens des animaux ramassés en 1986.

\section{Mortalité des reproducteurs}

En 1983, la mortalité, nulle avant la reprise de l'activité reproductrice, augmente peu après les premières pontes de manière comparable pour les 3 populations d'es- cargots (tableau II). En 1986, une mortalité importante, indépendante de la taille des animaux $(P>0,05)$, est observée dès la mise en conditions de reproduction, avant toute activité reproductrice. La mortalité qui affecte ensuite les survivants au cours de la période de reproduction est comparable à ce qui est observé pour les échantillons de 1983. 

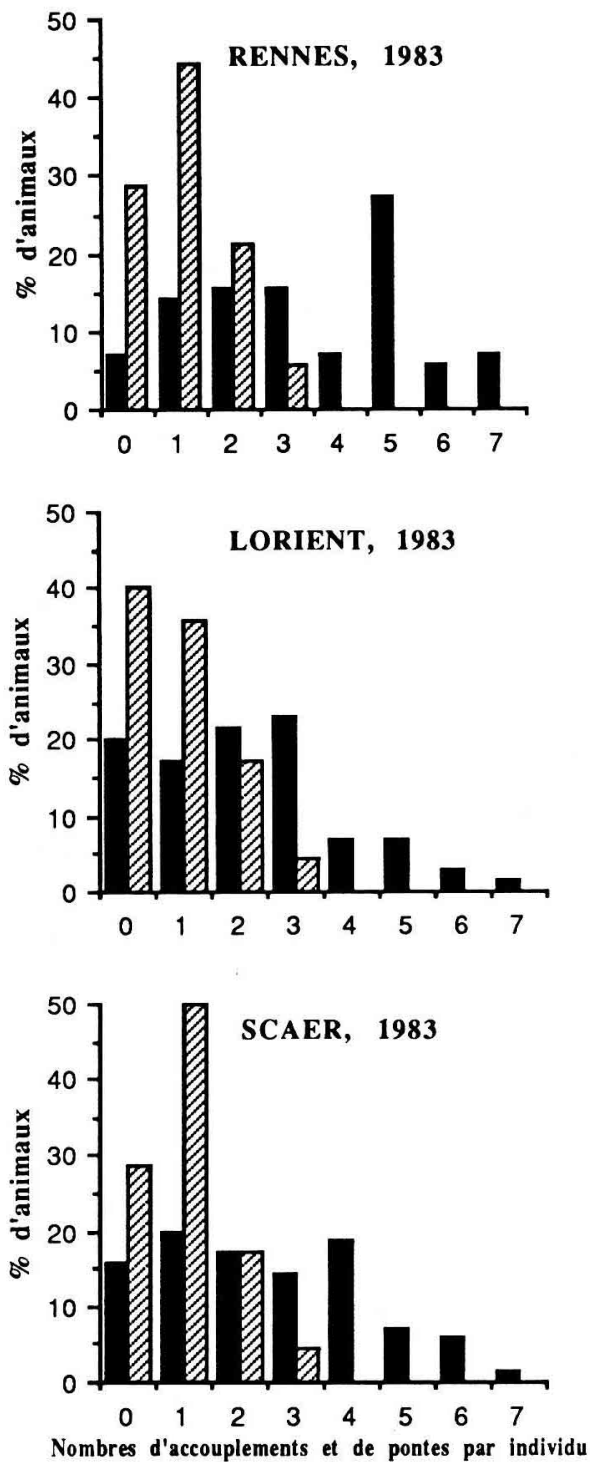

\section{DISCUSSION ET CONCLUSION}

Dans cette étude, les conditions d'environnement auxquelles sont soumis les escargots pendant la période de reproduction sont identiques pour tous les échantillons. Les différences enregistrées résultent
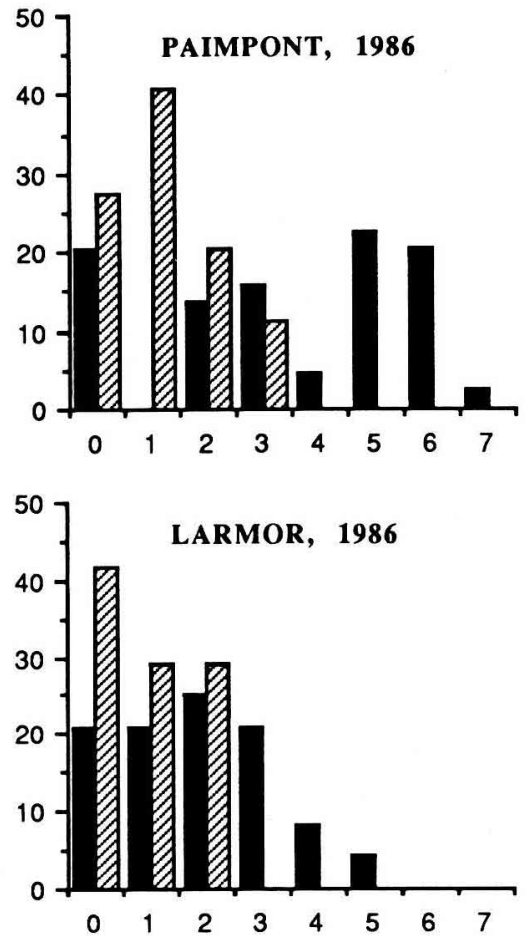

Fig 2. Courbes de fréquence des nombres d'accouplements $(\square)$ et de pontes (ש) par escargot, selon leur origine. Animaux placés en conditions artificielles pendant la période étudiée.

donc d'une éventuelle variation génétique ou de l'influence du milieu dans lequel ils se sont développés. Ces différences affectent l'activité reproductrice (chronologie, intensité) et la survie des animaux.

En 1983, seule l'existence d'un décalage de la reprise d'activité permet de diffé- 
rencier certains échantillons. Ainsi, les escargots originaires de Lorient se distinguent par leur précocité dans la reprise des pontes. Les données climatiques de cette station indiquent un écart thermométrique moyen de $1^{\circ} \mathrm{C}$ à partir du mois de janvier en faveur de Lorient, écart qui s'est élevé à plus de $3{ }^{\circ} \mathrm{C}$ en 1983 . Sachant qu'il existe une synchronisation très étroite entre la reprise de la reproduction des Gastéropodes pulmonés terrestres et les périodes favorables à cette activité (Peake, 1978), cette différence pourrait expliquer en partie la précocité de ces animaux. L'origine de ce phénomène peut toutefois être recherchée dans plusieurs directions. Ainsi, Cowie (1984) constate, chez Theba pisana Müller, que les individus de grande taille se distinguent par la précocité de leur reproduction or, les individus de Lorient ont en 1983 une taille significativement supérieure à celle des escargots de Rennes.

En 1986, la reprise de l'activité reproductrice n'intervient qu'après 5 semaines dans les 2 échantillons et les premières pontes n'ont lieu qu'après 9 semaines pour les individus originaires de Paimpont, 13 semaines pour ceux de Larmor. Rappelons que l'hiver 1985-1986 a été particulièrement rude (moyenne des températures minimales de février égale à $-2^{\circ} \mathrm{C}$ à Lorient et à $-3^{\circ} \mathrm{C}$ à Rennes) et que cette rigueur s'est prolongée tard dans la saison. Si l'on se réfère aux travaux d'Aupinel (comm pers), il est possible que le temps de latence observé dans la reprise de la reproduction soit lié à cette hibernation sévère. II en est probablement de même pour la mortalité enregistrée pendant les 5 semaines précédant la reproduction.

Les variations interannuelles agissent donc sur la mise en route de l'activité reproductrice : leur importance peut être considérable pour le devenir d'une popula- tion puisqu'elles sont susceptibles de conduire à son extinction (Sacchi, 1971).

L'intensité de l'activité reproductrice est également variable. Ainsi, les escargots originaires de Rennes et Paimpont s'accouplent plus souvent que ceux des 2 autres stations. Ce sont pourtant eux qui, au cours des 2 années, présentent le plus faible nombre d'œufs par ponte, mais ce phénomène peut être expliqué par les raisons suivantes :

- la taille des pontes est étroitement liée à la taille des individus;

- le nombre d'œufs des premières pontes ou pontes uniques est plus élevé que celui des pontes suivantes, or ces dernières sont plus nombreuses dans les échantillons de Rennes et surtout Paimpont.

L'année de la collecte a également une influence sur cette variable puisque la taille des pontes des escargots ramassés en 1986 est toujours inférieure à celle attendue d'après les résultats de 1983. Un phénomène identique est signalé par Le Calve (comm pers) pour des Helix aspersa originaires des Charentes.

Les variations affectant le nombre de pontes et le nombre d'œufs par ponte conduisent en fait à une homogénéisation du nombre total d'œufs par individu ayant pondu au bout d'une saison de reproduction, ce qui signifie une fécondité semblable de tous les échantillons, dans nos conditions d'étude. Un phénomène analogue a pu être observé entre individus d'une même population naturelle (celle de Rennes) mais dont la reproduction s'est déroulée, pour les uns, en conditions climatiques naturelles et pour les autres, dans les conditions artificielles identiques à celles du présent travail (Madec, 1989). $\mathrm{Au}$ total, les escargots d'une même région, en l'occurrence la Bretagne, ne semblent pas dotés de capacités reproductrices différentes. 
En résumé, à l'échelle régionale, les variations observées, qu'elles concernent un caractère morphologique ou physiologique, semblent dépendre autant de la dimension temporelle (année de la collecte) que de la dimension spatiale (localisation géographique) isolant les groupes d'individus. Une étude sur plusieurs années consécutives montre en effet que certaines variations adaptent l'individu (ou la génération) à un changement temporaire des conditions de l'environnement.

\section{RÉFÉRENCES}

Chevallier $H$ (1980) Les escargots du genre Helix commercialisés en France. Haliotis 10 , 11-24

Cowie RM (1984) The life-cycle and productivity of the land snail Theba pisana (Mollusca: Helicidae). J Anim Ecol 53, 311-325

Crook SJ (1980) Studies on the ecological genetics of Helix aspersa. Ph D Thesis, Univ of Dundee, $358 \mathrm{p}$

Daguzan J (1981) Contribution à l'élevage de l'escargot petit-gris Helix aspersa Müller. 1. Reproduction et éclosion des jeunes en bâtiment et en conditions thermographiquement contrôlées. Ann Zootech 30, 249-272
Ingram WM (1946) The european brownsnail in Oakland, California. Bull South Calif Acad Sci 45, 152-159

Madec $L$ (1987) Étude de la différenciation de quelques populations géographiquement séparées de l'espèce Helix aspersa Müller (Mollusque Gastéropode pulmoné) : aspects morphologiques, écophysiologiques et biochimiques. Thèse Doct Univ Rennes I, Sci Biol, $380 p$

Madec L, Daguzan J (1987) Étude de quelques facteurs affectant la reproduction de l'escargot petit-gris Helix aspersa Müller. Haliotis $16,77-91$

Moulin D (1980) Observations sur la reproduction en élevage de l'escargot petit-gris (Helix aspersa) en pays océanique (Normandie). Haliotis 10, 95-99

Peake J (1978) Distribution and Ecology of the Stylommatophora. In: Pulmonates (Fretter V, Peake J, eds) Acad Press, London, Vol 2A, 429-526

Potts DC (1975) Persistence and extinction of local populations of the garden snail Helix aspersa in unfavorable environments. Oecologia 21, 313-334

Sacchi C (1958) Les Mollusques terrestres dans le cadre des relations biogéographiques entre l'Afrique du Nord et l'Italie. Vie et Milieu 9, 11-52

Sacchi C (1971) Écologie comparée des Gastéropodes pulmonés des dunes méditerranéennes et atlantiques. Natura 62, 277-358 